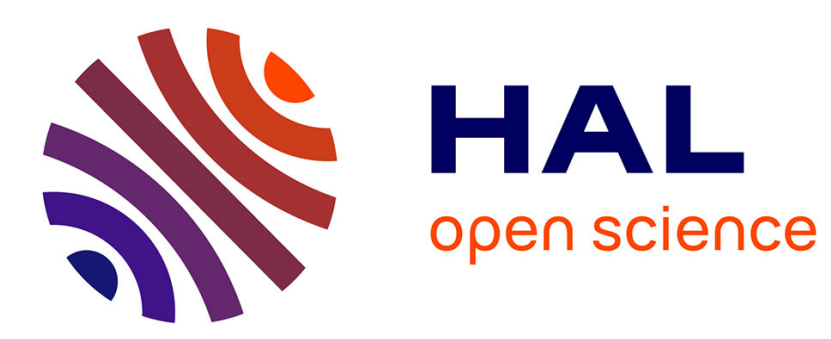

\title{
La valorisation salariale et professionnelle de la formation en entreprise diffère-t-elle selon le sexe? : l'exemple canadien
}

Nathalie Havet

\section{To cite this version:}

Nathalie Havet. La valorisation salariale et professionnelle de la formation en entreprise diffère-t-elle selon le sexe? : l'exemple canadien. 2006. halshs-00142878

\section{HAL Id: halshs-00142878 \\ https://shs.hal.science/halshs-00142878}

Submitted on 23 Apr 2007

HAL is a multi-disciplinary open access archive for the deposit and dissemination of scientific research documents, whether they are published or not. The documents may come from teaching and research institutions in France or abroad, or from public or private research centers.
L'archive ouverte pluridisciplinaire HAL, est destinée au dépôt et à la diffusion de documents scientifiques de niveau recherche, publiés ou non, émanant des établissements d'enseignement et de recherche français ou étrangers, des laboratoires publics ou privés. 


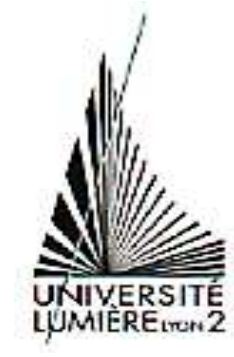

DOCUMENTS DE TRAVAIL - WORKING PAPERS

W.P. 06-02

\section{La valorisation salariale et professionnelle de la formation en entreprise diffère-t-elle selon le sexe ? : l'exemple canadien}

Nathalie Havet

Mars 2006

GATE Groupe d'Analyse et de Théorie Économique UMR 5824 du CNRS

93 chemin des Mouilles - 69130 Écully - France

B.P. $167-69131$ Écully Cedex

Tél. +33 (0)4 72866060 - Fax +33 (0)4 72866090

Messagerie électronique gate@gate.cnrs.fr

Serveur Web : www.gate.cnrs.fr 


\title{
La valorisation salariale et professionnelle de la formation en entreprise diffère-t-elle entre sexes ? : l'exemple canadien*
}

\author{
Nathalie HAVET ${ }^{\dagger}$ \\ GATE, Université Lumière, Lyon 2 (France)
}

\begin{abstract}
Résumé
L'objet de cet article est d'évaluer la valorisation salariale et professionnelle des formations continues, formelles et informelles, en centrant l'analyse sur les différences entre sexes. Pour ce faire, les données canadiennes de l'Enquête sur le milieu de travail et les employés (EMTE) 19992000 sont utilisées. On estime, par maximum de vraisemblance simulé, un probit trivarié récursif qui modélise simultanément la probabilité de suivre les différentes formations en entreprise et leur influence sur l'obtention d'une promotion. Les rendements salariaux des formations en entreprise et la rentabilité des promotions sont évalués à l'aide d'équation de salaires où ces variables sont instrumentées.
\end{abstract}

JEL : J16, J24, J31, C35.

\footnotetext{
*L'auteure tient à remercier Statistique Canada pour la mise à disposition de l'enquête EMTE et les gestionnaires de l'accès à distance de l'enquête EMTE et plus particulièrement Tony Fang, Anick Jonhson et Lucy Chung. L'auteure remercie aussi Guy Lacroix pour ses précieuses suggestions et remarques. Les opinions exprimées et les analyses développées dans cet article restent néanmoins propres à l'auteure.

${ }^{\dagger}$. Courriel : havet@gate.cnrs.fr
} 


\section{Introduction}

Du point de vue de l'employeur, la formation continue est une stratégie essentielle en matière de ressources humaines. Elle permet de pallier les déficits en compétences de base des individus, d'adapter les qualifications spécifiques des travailleurs à leurs besoins de court terme et de préparer les salariés aux profonds changements technologiques. A travers leurs actions de formation, les entreprises visent à améliorer l'efficacité productive de leur organisation. D'ailleurs, la plupart des études empiriques menées concluent à un effet positif et significatif de la formation continue sur les profits [Delame \& Kramarz (1997), OCDE (1995), Gauron (2000)].

La formation continue en entreprise devrait néanmoins aussi avoir des retombées positives au niveau individuel. En contribuant à une meilleure adaptation des salariés à leurs postes de travail, à un élargissement de leurs compétences productives et à une plus grande polyvalence des travailleurs, la formation continue en entreprise est censée favoriser la progression professionnelle des bénéficiaires, c'est-à-dire l'accès à une promotion et l'évolution de leur qualification. Ainsi, la formation continue en entreprise est un investissement pour les salariés [Becker (1964)] qui devrait au final avoir une incidence positive sur leur niveau de rémunération.

C'est à l'analyse de cet impact des actions de formation en entreprise sur les gains et les perspectives professionnelles des salariés qu'est consacré cet article. Néanmoins, cette question sera traitée en envisageant une dimension supplémentaire : les différences de valorisation de la formation continue entre sexes. On cherchera à savoir si les hommes et les femmes ont un accès différencié à la formation en entreprise et si celle-ci joue le même rôle pour les deux sexes en termes d'augmentations salariales et de promotions.

Les études empiriques antérieures ont obtenu des résultats mitigés. Premièrement, il n'y a pas de parfait consensus sur l'existence de différences d'investissement en formation en entreprise entre sexes. Il a été montré à plusieurs reprises sur données individuelles que les femmes avaient moins de chances d'acquérir une formation en entreprise que leurs homologues masculins et que cette différence pouvait expliquer une proportion importante de l'écart salarial entre sexes [Corcoran \& Duncan (1979), Duncan \& Hoffman (1979), Gronau (1988), Lynch (1992), Hill (2001), Olsen \& Sexton (1996) et Royalty (1996)]. Néanmoins, les travaux de Lillard \& Tan (1992), de Veum (1996) et très récemment de Turcotte, Léonard \& Montmarquette (2003) sur données canadiennes sont contradictoires puisqu'ils concluent à aucune différence entre sexes dans la probabilité de recevoir une formation en entreprise ou dans les heures de formation reçues.

Deuxièmement, les études analysant les différences de promotion entre sexes peuvent être classées en deux catégories : d'une part celles basées sur des données spécifiques à une seule entreprise (institution financière, grande compagnie d'assurance, etc) ou à un secteur particulier (avocats, services de santé, chercheurs universitaires, etc) et d'autre part celles plus récentes et moins nombreuses basées sur des panels de salariés représentatifs de l'ensemble des professions. Les résultats obtenus à partir de la première série d'études sont très hétérogènes. Certes, un certain nombre concluent qu'à compétences égales, les femmes ont des probabilités plus faibles d'être promues par rapport aux hommes [Cabral, Ferber \& Green (1981), Cannings (1988), DiPrete \& Soule (1988), Spurr (1990), Long, Allison \& McGinnis (1993), Spurr \& Sueyoshi (1994), Paulin \& Mellor (1996), Pudney \& Shields (2000)]. Mais d'autres indiquent que la variable de sexe n'a aucune influence sur les chances de promotion [Lewis (1986), Hartman (1987), Laband \& Lentz (1993)] ou même trouvent que les 
femmes sont significativement plus promues que les hommes [Stewart \& Gudykunst (1982), Gerhart \& Milkovich (1989), Shenhav (1992), Powell \& Butterfield (1994), Hersch \& Viscusi (1996)]. La diversité de ces résultats est sans aucun doute à mettre au compte de la particularité des données et des différences dans les méthodes économétriques utilisées. Les études sur panels de salariés représentatifs présentent des conclusions plus robustes. Elles trouvent des taux de promotion plus faibles pour les femmes [Olson \& Becker (1983), McCue (1996), Pargamit \& Veum (1999), CobbClark (2001), Groot \& Maassen von den Brink (1996)] à l'exception de Booth, Francesconi \& Frank (1998) sur données anglaises.

Enfin, seulement quelques articles ont montré que la formation en entreprise est la clé de l'accès à la promotion et encore le lien mis en évidence n'est pas immédiat [Béret \& Dupray (1998), Michaudon (2000)].

Une des originalités de notre étude empirique est d'appréhender toutes ces questions simultanément. En outre, l'utilisation de l'Enquête sur le milieu de travail et les employés 1999-2000 nous offre un nouvel éclairage sur la situation canadienne. En dépit de travaux réalisés au Canada sur les déterminants d'une participation à une formation continue, peu se sont intéressés à son impact en termes de progression de carrières. L'EMTE a deux aspects séduisants qui sont exploités ici. D'une part, elle interrelie des renseignements recueillis auprès des établissements et auprès des travailleurs permettant de bien isoler les caractéristiques de l'entreprise au sein de laquelle les salariés formés et/ou promus travaillent et donc de déterminer précisément les effets propres des caractéristiques des travailleurs et notamment les différences hommes/femmes. D' autre part, l'EMTE distingue la formation en cours d'emploi de la formation structurée, ce qui offre la possibilité d'examiner les liens entre formation continue formelle et informelle, de comparer leurs impacts respectifs sur les carrières et de savoir s'il existe des différences entre sexes dans la nature et les rendements des formations suivies. Jusque-là, la plupart des études se sont uniquement concentrées sur la formation structurée, faute de données disponibles sur les autres catégories de formations en entreprise.

Les rendements de la formation en entreprise et la rentabilité des promotions - et leurs écarts entre sexes - sont évalués à l'aide d'équations de salaires où les variables de formation et de promotion sont instrumentées en estimant des probits multivariés récursifs qui modélisent simultanément la probabilité de suivre les différentes formations en entreprise et leur influence sur l'obtention d'une promotion.

La suite de cet article est organisé comme suit. La section 2 présente les données de l'Enquête sur le milieu de travail et les employés (EMTE). La section 3 détaille la méthode économétrique choisie pour mesurer la valorisation salariale et professionnelle de la formation continue. Enfin, les résultats des estimations seront commentés dans la section 4.

\section{Les données}

\subsection{L'Enquête sur le milieu du travail et les employés}

Nous utilisons les données 1999-2000 de l'Enquête sur le milieu du travail et les employés (EMTE) qui a été réalisée par Statistique Canada. L'EMTE comporte deux volets : 1) une enquête

auprès des établissements sur leurs caractéristiques, leurs changements organisationnels, la formation 
et autres pratiques en matière de ressources humaines et de stratégies d'entreprises ; 2) une enquête auprès des employés dans ces milieux de travail afin de recueillir des données sur leurs salaires, leurs heures de travail, leur capital humain, les caractéristiques de leur emploi et autres informations démographiques habituellement recueillies dans les enquêtes auprès des ménages (âge, situation matrimoniale, niveau de scolarité, etc). Il en résulte une base de données riche de renseignements interreliés sur les milieux de travail et les employés.

Un autre avantage de l'EMTE est qu'elle comprend des informations précises sur la formation en entreprise. Dans le questionnaire adressé aux employés, il leur est demandé si au cours des 12 derniers mois, ils ont d'une part suivi « des cours de formation structurée liée à leur emploi » et d'autre part s'ils ont reçu « une formation en cours d'emploi », les deux étant parrainées par l'employeur. La formation structurée désigne les activités de formation ayant un format prédéterminé, des objectifs prédéfinis, un contenu précis et dont le progrès peut être contrôlé ou évalué. Elle s'apparente ainsi à de la formation formelle. En revanche, la formation en cours d'emploi n'est pas définie dans le questionnaire mais on peut considérer qu'une part importante correspond à de la formation informelle puisqu'elle est généralement acquise par auto-apprentrissage $(9,4 \%)$ ou à l'aide de collègues de travail $(29,3 \%)$ ou de superviseurs $(28,7 \%)$. Au contraire, $83 \%$ de la formation structurée est donnée par un formateur interne ou externe. Un aspect séduisant de l'EMTE réside ainsi dans la possibilité d'examiner les liens entre formations formelle et informelle et de savoir s'il existe des différences entre sexes dans la nature des formations suivies.

Seuls les individus qui étaient actifs en 1999 et 2000, soit 18870 employés dont $43 \%$ de femmes, sont retenus pour cette étude empirique. Le tableau 1 présente les statistiques descriptives de notre échantillon, en distinguant les deux sexes.

\subsection{Formation structurée, formation en cours d'emploi, promotion et salaires}

Selon les réponses apportées par les employés, un peu plus de la moitié d'entre eux (54\%) ont suivi une formation en entreprise du 1er avril 1999 au 31 mars 2000. La proportion d'employés ayant suivi une formation structurée est de l'ordre de $36 \%$ contre $31 \%$ pour celle de la formation en cours d'emploi. On observe que $13 \%$ des employés ont acquis une combinaison des deux types de formation au cours de la même année (Tableau 2).

La proportion de femmes ayant suivi une formation en entreprise est très légèrement supérieure à celle des hommes $(54,2 \%$ contre 53,6\%). La différence entre sexes est principalement due à un pourcentage plus élevé de femmes ayant reçu une formation en cours d'emploi (32,6\% contre 29,6\% pour les hommes). Il est à noter que les femmes ont moins suivi une formation structurée seule ; celle-ci est davantage couplée avec une formation en cours d'emploi.

Le tableau 3 montre que la formation en entreprise favorise la progression des carrières. La participation à un programme de formation continue accroît sensiblement les chances d'obtenir une promotion au cours de la même année : seulement $13 \%$ des individus n'ayant pas suivi de formation sont promus, contre $19 \%$ pour ceux ayant suivi une formation structurée, et $25 \%$ pour ceux ayant suivi une formation en cours d'emploi. Dans le questionnaire de l'EMTE, le terme promotion désigne « un changement de fonctions ou responsabilités qui entraîne une augmentation à la fois du salaire et de la complexité ou des responsabilités du poste ». Par conséquent, la formation en entreprise semble bien avoir des répercussions en termes de postes occupés dans la hiérarchie et de progression salariale. 


\begin{tabular}{|c|c|c|c|}
\hline & ENSEMBLE & HOMMES & FEMMES \\
\hline Age moyen (2000) & 40,4 & 40,6 & 40,3 \\
\hline $\begin{array}{l}\text { Nombre moyen d'années d'expérience } \\
\text { de travail à temps plein }\end{array}$ & 17,1 & 18,8 & 15,5 \\
\hline $\begin{array}{l}\text { Nombre moyen de mois d'ancienneté } \\
\text { avec l'employeur actuel (2000) }\end{array}$ & 109,0 & 116,3 & 102,3 \\
\hline \multicolumn{4}{|l|}{ Heures travaillées } \\
\hline Moins de 30 heures/semaine & $18,5 \%$ & $13,6 \%$ & $22,9 \%$ \\
\hline Entre 30 et 50 heures/semaine & $77,3 \%$ & $79,0 \%$ & $75,7 \%$ \\
\hline 50 heures et plus/semaine & $4,2 \%$ & $7,4 \%$ & $1,4 \%$ \\
\hline \multicolumn{4}{|l|}{ Statut d'emploi } \\
\hline Emploi non permanent & $7,6 \%$ & $6,6 \%$ & $8,5 \%$ \\
\hline Emploi de supervision & $38,7 \%$ & $45,1 \%$ & $32,8 \%$ \\
\hline Emploi syndiqué & $29,6 \%$ & $31,7 \%$ & $27,7 \%$ \\
\hline $\begin{array}{l}\text { Emploi avec procédure } \\
\text { d'évaluation du rendement }\end{array}$ & $56,8 \%$ & $54,3 \%$ & $59,1 \%$ \\
\hline \multicolumn{4}{|l|}{ Niveau de scolarité } \\
\hline Pas de diplôme d'études secondaires & $10,9 \%$ & $12,7 \%$ & $9,2 \%$ \\
\hline Diplômes d'études secondaires & $19,5 \%$ & $19,8 \%$ & $19,5 \%$ \\
\hline Certificat & $10,6 \%$ & $14,2 \%$ & $7,3 \%$ \\
\hline Diplôme collégial & $28,6 \%$ & $23,2 \%$ & $33,6 \%$ \\
\hline Diplôme universitaire & $30,2 \%$ & $30,0 \%$ & $30,4 \%$ \\
\hline Formation & $53,9 \%$ & $53,6 \%$ & $54,2 \%$ \\
\hline Proportion suivant une formation structurée & $35,9 \%$ & $36,7 \%$ & $35,2 \%$ \\
\hline Proportion suivant une formation en cours d'emploi & $31,2 \%$ & $29,6 \%$ & $32,6 \%$ \\
\hline Proportion ayant eu une promotion & $18,5 \%$ & $18,6 \%$ & $18,3 \%$ \\
\hline Salaire horaire moyen en 2000 (en $\$$ ) & 20,0 & 22,2 & 17,3 \\
\hline Nombre d'observations & 18870 & 10756 & 8114 \\
\hline
\end{tabular}

TAB. 1: STATISTIQUES DESCRIPTIVES

\begin{tabular}{lcccc}
\hline & $\begin{array}{c}\text { Uniquement } \\
\text { Formation structuree }\end{array}$ & $\begin{array}{c}\text { Uniquement } \\
\text { Formation en cours d'emploi }\end{array}$ & $\begin{array}{c}\text { Les deux types } \\
\text { de formation }\end{array}$ & $\begin{array}{c}\text { Aucune } \\
\text { formation }\end{array}$ \\
\hline Ensemble & $22,76 \%$ & $17,97 \%$ & $13,18 \%$ & $46,09 \%$ \\
Hommes & $24,00 \%$ & $16,86 \%$ & $12,74 \%$ & $46,39 \%$ \\
Femmes & $21,62 \%$ & $18,98 \%$ & $13,58 \%$ & $45,82 \%$ \\
\hline
\end{tabular}

TAB. 2: TAUX D’ ACCÈs À UNE FORMATION CONTINUE ENTRE LE 1ER AVRIL 1999 ET LE 31 MARS 2000, EN FONCTION DU SEXE

Dans leur ensemble, les hommes et les femmes ont des taux de promotion identiques $(18,5 \%)$. Toutefois, cette situation globale cache des disparités entre sexes dans la valorisation professionnelle de chaque type de formation continue. Par exemple, les femmes ayant suivi une formation en cours d'emploi ont des taux de promotion inférieurs de 5 points à ceux de leurs homologues masculins. En revanche, les femmes n'ayant pas suivi de formation en entreprise ont davantage de chances d'ob- 


\begin{tabular}{lccccc}
\hline & Général & $\begin{array}{c}\text { Uniquement } \\
\text { Formation structurée }\end{array}$ & $\begin{array}{c}\text { Uniquement } \\
\text { Formation en cours d'emploi }\end{array}$ & $\begin{array}{c}\text { Les deux types } \\
\text { de formation }\end{array}$ & $\begin{array}{c}\text { Aucune } \\
\text { formation }\end{array}$ \\
\hline Ensemble & $18,45 \%$ & $19,40 \%$ & $24,93 \%$ & $25,10 \%$ & $13,56 \%$ \\
Hommes & $18,59 \%$ & $19,52 \%$ & $27,66 \%$ & $27,75 \%$ & $12,29 \%$ \\
Femmes & $18,34 \%$ & $19,29 \%$ & $22,73 \%$ & $22,84 \%$ & $14,73 \%$ \\
\hline
\end{tabular}

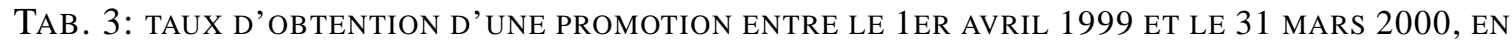
FONCTION DU SEXE ET DES FORMATIONS EN ENTREPRISE SUIVIES

tenir une promotion que les hommes dans la même situation. Au vu de ces statistiques descriptives, l'absence de formation continue semble être davantage un handicap dans les carrières des hommes que dans celles des femmes. Toutefois, ce résultat pourrait simplement refléter que les hommes et les femmes ne participant pas au programme de formation ne sont pas deux groupes homogènes. Au contraire, ils pourraient se distinguer par certaines caractéristiques individuelles.

Le tableau 4 montre que les salaires horaires sont plus élevés dans les groupes des hommes formés, en particulier ceux ayant suivi une formation structurée. De même, les individus ayant bénéficié d'une promotion au cours de l'année ont un salaire horaire moyen supérieur à ceux qui n'ont pas été promus. Il semble néanmoins que ce soit davantage la participation à un programme de formation structurée que la promotion qui soit la clé d'une forte rémunération.

\begin{tabular}{llccc}
\hline & & Formation structurée & Formation en cours d'emploi & Aucune formation \\
\hline \multirow{6}{*}{ Promus } & Ensemble & $22,50 \$$ & $20,91 \$$ & $17,65 \$$ \\
& Hommes & $24,97 \$$ & $23,22 \$$ & $20,10 \$$ \\
& Femmes & $20,22 \$$ & $19,00 \$$ & $15,39 \$$ \\
\hline \multirow{2}{*}{ Non promus } & Ensemble & $23,07 \$$ & $21,00 \$$ & $18,75 \$$ \\
& Hommes & $25,89 \$$ & $23,56 \$$ & $23,35 \$$ \\
& Femmes & $20,15 \$$ & $18,43 \$$ & $15,21 \$$ \\
\hline & Ensemble & $22,35 \$$ & $20,88 \$$ & $17,47 \$$ \\
& Hommes & $24,63 \$$ & $23,08 \$$ & $19,64 \$$ \\
& Femmes & $20,23 \$$ & $19,17 \$$ & $15,42 \$$
\end{tabular}

TAB. 4: SALAIRE HORAIRE MOYEN AU 31 MARS 2000, EN FONCTION DU SEXE, DES FORMATIONS EN ENTREPRISE SUIVIES ET DES PROMOTIONS REÇUES

On peut aussi noter que les écarts salariaux entre sexes sont plus prononcés parmi les salariés promus. Par exemple, pour les individus n'ayant suivi aucune formation continue mais ayant obtenu une promotion, la différence de salaire hommes/femmes est de près de $53 \%$ du salaire féminin contre $27 \%$ sur l'ensemble de l'échantillon. Ce résultat «brut »ne suffit pas pour conclure définitivement que le processus de promotion exacerbe les disparités salariales entre sexes. Il ne tient pas compte des effets conjoints des autres variables individuelles (poste, diplôme, etc) sur l'accès à la formation, l'obtention d'une promotion et les salaires. D'où l'importance de recourir à une modélisation économétrique pour avoir une comparaison fiable des rendements des formations continues et des promotions, toutes choses étant égales par ailleurs. En tout cas, ces premiers résultats mettent en évidence qu'il est indispensable de distinguer les différents types de formation continue car leurs impacts en termes de carrières semblent être bien différents. 


\section{Spécification économétrique}

Notre but est d'avoir une spécification économétrique qui modélise l'incidence de la formation en entreprise à la fois sur les salaires et à la fois sur les promotions. Le cadre généralement retenu pour évaluer l'impact de la formation continue sur les carrières salariales est une équation de salaire mincérienne incluant une dichotomique qui vaut 1 si l'individu a suivi une formation et 0 sinon. Or, il est très probable que chaque travailleur décide de son investissement en formation continue en fonction de son rendement anticipé en termes de surcroît de salaire (direct ou indirect via l'obtention d'une promotion). Les variables de formation et de promotion sont donc endogènes et doivent ainsi être "instrumentées" afin d'éviter que les évaluations soient biaisées. Or, les diverses décisions conduisant à former un salarié et éventuellement à le promouvoir, se prennent souvent de manière concomitante. Il est, par conséquent, nécessaire de tenir compte de cette potentielle simultanéité dans les investissements en formation et le processus de promotion. C'est pourquoi, dans une première étape, on estime un probit trivarié récursif qui permet de modéliser simultanément les probabilités de suivre chaque type de formation (structurée ou en cours d'emploi) et leur influence sur l'obtention d'une promotion. Dans une seconde étape, on estime une équation de salaire mincérienne où ces probabilités prédites sont introduites afin de pallier au caractère endogène de ces variables.

\subsection{Probit trivarié récursif : Valorisation professionnelle des formations continues}

La valorisation professionnelle des deux types de formation continue est estimée à l'aide d'un probit trivarié récursif. D'une part, il permet de tenir compte du fait que les formations en entreprise peuvent être un élément prépondérant voire une condition préalable à une promotion.

D'autre part, il modélise toutes les corrélations possibles entre les types de formation et les promotions, reflets d'éventuelles simultanéités dans les décisions. Par exemple, ce modèle offre l'avantage de prendre en compte le fait que les salariés peuvent combiner ou non la participation à une formation structurée et à une formation en cours d'emploi, via l'estimation d'un coefficient de corrélation $\rho_{s e}$ entre les résidus de ces deux équations de participation. Puisque dans les modèles à variables discrètes, des problèmes de cohérence logique [Maddala (1983)] rendent difficiles l'expression d'une simultanéité directe des décisions, on en tient compte ici par les coefficients de corrélation entre les résidus, qui mesurent la corrélation entre les caractéristiques non observables des salariés ${ }^{2}$. La décision de suivre une formation structurée et celle de suivre une formation en cours d'emploi peuvent être corrélées pour plusieurs raisons. La corrélation peut être positive si des liens de complémentarité existe entre les deux décisions, reflétant par exemple une volonté commune -mais inobservée- d'investir en capital humain. Mais la corrélation peut aussi être négative si les deux types de formation sont plutôt des substituts, constituant des outils différents permettant d'acquérir des connaissances similaires. Par ailleurs, on peut imaginer que certaines caractéristiques individuelles inobservables tels que l'habileté, détermination ou envie de réussir du salarié influencent à la fois ses chances de participer à une formation continue et celles d'obtenir une promotion, d'où des corrélations potentielles entre ces variables $\left(\rho_{s p}, \rho_{e p}\right)$.

\footnotetext{
${ }^{2}$ En fait, le modèle économétrique retenu n'offre pas la possibilité de rendre la décision de suivre une formation directement de l'autre, car les données dont on dispose et en particulier le manque d'information sur la chronologie des formations suivies ne nous permettent pas de déterminer quelle formation influence laquelle. Or l'expression d'une simultanéité directe des décisions de formation exige que cette question soir tranchée. En revanche, comme l'on suppose que la formation est une condition préalable à une promotion, on a pu introduire comme déterminant de l'obtention d'une promotion les variables de participations à une formation en entreprise.
} 
Plus précisément, le cadre formel de notre spécification est le suivant :

$$
\begin{gathered}
y_{i s}=\left\{\begin{array}{lll}
1 & \text { si } & y_{i s}^{*}=Z_{i j} \gamma_{s}+u_{i s}>0 \\
0 & \text { si } & y_{i s}^{*}=Z_{i j} \gamma_{s}+u_{i s} \leqslant 0,
\end{array}\right. \\
y_{i e}=\left\{\begin{array}{lll}
1 & \text { si } & y_{i e}^{*}=Z_{i j} \gamma_{e}+u_{i e}>0 \\
0 & \text { si } & y_{i e}^{*}=Z_{i j} \gamma_{e}+u_{i e} \leqslant 0,
\end{array}\right. \\
y_{i p}=\left\{\begin{array}{lll}
1 & \text { si } y_{i p}^{*}=y_{i s} \alpha_{s}+y_{i e} \alpha_{e}+V_{i j} \gamma_{p}+u_{i p}>0 \\
0 & \text { si } & y_{i p}^{*}=y_{i s} \alpha_{s}+y_{i e} \alpha_{e}+V_{i j} \gamma_{p}+u_{i p} \leqslant 0,
\end{array}\right.
\end{gathered}
$$

où les résidus $\left(u_{i s}, u_{i e}, u_{i p}\right)$ suivent une distribution normale jointe trivariée :

$$
\left(\begin{array}{l}
u_{i s} \\
u_{i e} \\
u_{i p}
\end{array}\right) \sim \mathcal{N}\left[\left(\begin{array}{l}
0 \\
0 \\
0
\end{array}\right),\left(\begin{array}{ccc}
1 & \rho_{s e} & \rho_{s p} \\
\rho_{s e} & 1 & \rho_{e p} \\
\rho_{s p} & \rho_{e p} & 1
\end{array}\right)\right]
$$

Les équations (1) et (2) modélisent respectivement la décision du salarié de suivre une formation structurée $\left(y_{i s}=1\right)$ et celle de suivre une formation en cours d'emploi $\left(y_{i e}=1\right)$. On retient les mêmes déterminants $Z$ pour les deux types de formation mais leur influence peut différer selon le type de formation ( $\gamma_{s}$ a priori différent de $\left.\gamma_{e}\right)$. L'équation (3) modélise la probabilité d'être promu $\left(y_{i p}=1\right)$. $\alpha_{s}$ et $\alpha_{e}$ captent respectivement l'incidence de la formation structurée et de la formation en cours d'emploi. Si la formation en entreprise est un élément clé de l'obtention d'une promotion, ces deux paramètres devraient être positifs.

Certaines contraintes d'identification doivent être imposées afin d'estimer tous les paramètres. Comme dans tout modèle probit, les variances des résidus ne sont pas identifiables et par conséquent la première restriction consiste à les normaliser à 1 [voir équation (4)]. La seule autre restriction est exigée par la récursivité du modèle. Les résidus des équations latentes n'étant pas indépendants, les paramètres de l'équation de promotion ne peuvent pas être identifiés si les déterminants des promotions $V_{i j}$ incluent tous les déterminants des formations $Z_{i j}{ }^{3}$. La contrainte d'identification est qu'au moins une variable explicative des décisions de formations soit exclue de l'équation de promotion ${ }^{4}$.

La log-vraisemblance associée à ce modèle a pour expression :

$$
\ln \mathcal{L}=\sum_{i=1}^{N} \sum_{j=0}^{1} \sum_{k=0}^{1} \sum_{l=0}^{1} h_{i}(j, k, l) \ln \left[P\left(y_{i s}=j, y_{i e}=k, y_{i p}=l\right)\right],
$$

où

$$
h_{i}(j, k, l)=\left\{\begin{array}{cc}
1 & \text { si } \\
0 & \text { sinon }
\end{array}\left(y_{i s}=j, y_{i e}=k, y_{i p}=l\right), j, k, l=0 \text { ou } 1 .\right.
$$

et par exemple

$$
\begin{aligned}
P\left(y_{i s}=0, y_{i e}=0, y_{i p}=0\right) & =\Phi_{3}\left(-Z \gamma_{s},-Z \gamma_{e},-V \gamma_{p}, \rho_{s e}, \rho_{s p}, \rho_{e p}\right) \\
& =\int_{-\infty}^{-Z \gamma_{s}} \int_{-\infty}^{-Z \gamma_{e}} \int_{-\infty}^{-V \gamma_{p}} \phi_{3}\left(u_{i s}, u_{i e}, u_{i p}, \rho_{s e}, \rho_{s p}, \rho_{e p}\right) \cdot d u_{i s} d u_{i e} d u_{i p},
\end{aligned}
$$

\footnotetext{
${ }^{3}$ Voir Maddala (1983, p.117-138) pour un survol des problèmes d'identification dans les modèles récursifs à équations simultanées.

${ }^{4}$ La variable "utilisation d'un ordinateur" est supposée influencer la participation à une formation mais pas directement l'obtention d'une promotion.
} 
avec $\phi_{3}$ et $\Phi_{3}$ les fonctions de densité et de répartition d'une loi normale trivariée standardisée. Les autres probabilités se calculent de la même manière.

Comme l'estimation exacte des paramètres par la méthode du maximum de vraisemblance nécessite le calcul d'intégrales triples, on a recours à une procédure de simulations, l'algorithme GewekeHajivassiliou-Keane (GHK), pour obtenir une approximation de ces intégrales et nos estimateurs sont les solutions de la maximisation de la log-vraisemblance simulée ${ }^{5}$ : les probabilités simulées remplacent les intégrales des lois normales multivariées dans la fonction de vraisemblance qui est alors maximisée en utilisant les techniques traditionnelles.

Puisque plusieurs travailleurs sélectionnés appartiennent au même établissement, certaines observations risquent d'être fortement corrélées entre elles. Ce problème est de nature à biaiser les estimateurs des variances des coefficients estimés. On y remédie en utilisant un estimateur de variance de type Huber-White.

Pour qu'une formation continue soit suivie, celle-ci doit être non seulement acceptée par le travailleur mais aussi proposée par l'entreprise. C'est pourquoi, on retient comme variables explicatives $Z$ de la participation à une formation non seulement des caractéristiques relatives au salarié (sexe, ancienneté, expérience, diplôme, type et statuts de l'emploi occupé) mais aussi certaines caractéristiques relatives à l'entreprise (taille, branche d'activité, région du siège social, caractère lucratif ou non, ses taux de roulement et d'emplois vacants). Des caractéristiques individuelles moins habituelles sont introduites telles que l'utilisation ou non par les travailleurs d'un ordinateur et le fait que leur rendement soit ou non évalué par une procédure courante (rapport écrit, rencontre personnelle avec un supérieur ou rapport normalisé). Il ne serait pas étonnant que les travailleurs utilisant un ordinateur soient plus susceptibles de suivre une formation, notamment portant sur l'apprentissage de logiciels. Les salariés dont le rendement est évalué par une procédure courante devrait avoir une probabilité plus élevée de suivre une formation en entreprise car leur acquisition de nouvelles connaissances - et donc leur surcroît de productivité - a plus de chances d'être reconnue et donc récompensée financièrement.

Selon les arguments théoriques traditionnels [Olson \& Becker (1983), Cobb-Clark (2001)], la probabilité qu'un individu soit promu dépend à la fois de la probabilité que l'entreprise décide de combler l'emploi vacant et de la probabilité que l'individu réponde à certains critères et accepte la promotion. Ainsi, on retient comme variables explicatives des promotions $V$ des caractéristiques relatives au salarié (capital humain, attachement au marché du travail, etc) affectant leur rendement anticipé et des caractéristiques relatives aux entreprises (occupations, secteur d'activité, syndicat, taux de roulement et d'emplois vacants) influençant la productivité attendue et la probabilité que l'entreprise décide de combler certains emplois. Afin de comparer la valorisation professionnelle des formations continues entre hommes et femmes, des variables croisées sexe*formation structurée et sexe *formation en cours d'emploi ont été introduites.

\footnotetext{
${ }^{5}$ L'algorithme de simulation GHK, utilisé pour estimer le probit trivarié, exploite le fait que toute fonction de distribution normale multivariée peut s'écrire comme le produit de fonctions de distribution univariées conditionnelles. Pour plus de détails, voir Greene (2003, p.931-933), Stern (2000), Geweke, Keane \& Runkle (1994). Le nombre de tirages pour les simulations est ici de 150, nombre suffisamment grand pour rendre négligeable l'erreur de simulations.
} 


\subsection{Equation de salaire mincérienne : Valorisation salariale des formations continues et des promotions}

Afin de mesurer le rendement des formations continues et des promotions, on introduit dans une équation de salaire classique à la Mincer (1974), les probabilités individuelles de suivre les deux types

de formation $\left[\hat{P}\left(y_{i s}=1\right), \hat{P}\left(y_{i e}=1\right)\right]$ et d'obtenir une promotion $\left[\hat{P}\left(y_{i p}=1\right)\right.$ ], estimées à l'aide du probit trivarié récursif précédent :

$$
\ln W_{i j}=X_{i j} \beta+\hat{P}\left(y_{i s}=1\right) \delta_{s}+\hat{P}\left(y_{i e}=1\right) \delta_{e}+\hat{P}\left(y_{i p}=1\right) \delta_{p}+\varepsilon_{i j} .
$$

D'après les statistiques descriptives, on peut s'attendre à ce que $\delta_{s}>\delta_{e}$ ou en d'autres termes que le rendement salarial de la formation structurée soit supérieur à celui de la formation en cours d'emploi. Cela indiquerait que plus un investissement en qualification est imparfaitement observé, plus son rendement en termes de rémunération est réduit. En effet, on peut supposer que les entreprises ont une information plus précise sur les connaissances acquises par un salarié lors d'une formation structurée (formation formelle) que lors d'une formation en cours d'emploi (formation informelle).

La variable dépendante utilisée est le log du salaire horaire au 31 mars 2000. On retient de nombreux déterminants dans l'équation de salaire tels que des variables reflétant le capital humain du salarié (ancienneté, expérience, diplôme), sa situation familiale (en couple, nombre d'enfants), le type d'emploi occupé (temps partiel, flexibilité des horaires, syndiqué, profession) et les caractéristiques de son établissement (branche d'activité, taille de l'entreprise, région du siège social). On ajoute à ces déterminants traditionnels d'autres variables relatives à la propriété étrangère et aux organismes à but non lucratif. Plusieurs études [Lipsey (1994), Aitken, Harrison \& Lipsey (1996), Feliciano \& Lipsey (1999), Drolet (2002)] ont mis en évidence que des niveaux salariaux plus élevés étaient associés aux entreprises détenues par des investisseurs étrangers. Selon Aitken et al. (1996), la différence de rémunération entre les entreprises étrangères et canadiennes ceteris paribus découlerait de différences de productivité liées notamment à un accès plus facile aux actifs financiers, à la technologie et à l'innovation. L'EMTE demande aux entreprises de faire état du pourcentage de leurs avoirs détenus par des intérêts étrangers. On considère ici que les entreprises sont étrangères si au moins $51 \%$ de leurs avoirs sont de propriété étrangère. De même, il a été noté à quelques reprises dans la littérature que les organismes sans but lucratif offrent en général des salaires plus faibles qu'ailleurs et en particulier pour les gestionnaires [Handy \& Katz (1998), Ruhm \& Borkoski (2001), Leete (2001)].

De nouveau, on utilise un estimateur de variance de type Huber-White pour tenir compte des éventuelles corrélations entre les observations des travailleurs d'un même établissement. En outre, on croise les variables de formations continues et de promotions avec le sexe afin de comparer leur valorisation salariale entre hommes et femmes.

\section{Les résultats empiriques}

Les coefficients estimés du probit récursif trivarié sont reportés au tableau 5 et ceux de l'équation de salaire au tableau 6. Dans les deux estimations, de nombreuses variables sont statistiquement significatives et ont l'effet attendu. Par exemple, les salariés embauchés dans les entreprises au taux de 
roulement les plus importants ont une probabilité plus élevée d'être promus. Les salaires sont croissants avec le niveau de scolarité, l'expérience professionnelle, l'ancienneté, la taille de l'établissement et le fait qu'il soit de propriété étrangère ${ }^{6}$. Il est à noter que l'on tient compte dans l'équation de salaire du nombre d'heures non rémunérées habituellement effectuées par semaine et qu'il a un impact significatif sur les salaires. Ces heures seraient donc compensées financièrement de façon indirecte. Par exemple, dans le cas d'individus payés selon un salaire annuel, une semaine de travail normale pourrait être de 40 heures, mais l'employeur pourrait s'attendre à ce que ses salariés travaillent des heures supplémentaires au besoin sans que celles-ci soient rémunérées. Le fait que l'individu accepte ce genre de conditions semble s'accompagner d'un salaire horaire plus élevé.

\begin{tabular}{|c|c|c|c|c|c|c|}
\hline \multirow{2}{*}{ Variables } & \multicolumn{2}{|c|}{$\begin{array}{l}\text { FORMATION } \\
\text { STRUCTURÉE }\end{array}$} & \multicolumn{2}{|c|}{$\begin{array}{c}\text { FORMATION } \\
\text { EN COURS D'EMPLOI }\end{array}$} & \multicolumn{2}{|c|}{ PROMOTION } \\
\hline & Param. & P. value & Param. & P. value & Param. & P. value \\
\hline Constante & $-1,011$ & $(0,000)$ & $-0,557$ & $(0,002)$ & $\begin{array}{l}-0,888 \\
\end{array}$ & $(0,000)$ \\
\hline Sexe $($ homme $=1)$ & 0,085 & $(0,144)$ & $-0,034$ & $(0,545)$ & $-0,125$ & $(0,083)$ \\
\hline Ancienneté avec l'employeur & 0,000 & $(0,595)$ & $-0,001$ & $(0,110)$ & $-0,003$ & $(0,004)$ \\
\hline Ancienneté au carré/100 & $-0,000$ & $(0,185)$ & $-0,000$ & $(0,073)$ & 0,000 & $(0,197)$ \\
\hline Expérience à temps plein & $-0,005$ & $(0,559)$ & 0,001 & $(0,857)$ & $-0,016$ & $(0,182)$ \\
\hline Expérience à temps plein au carré/100 & 0,011 & $(0,653)$ & $-0,032$ & $(0,096)$ & 0,028 & $(0,414)$ \\
\hline Emploi permanent & 0,125 & $(0,164)$ & $-0,022$ & $(0,811)$ & 0,006 & $(0,950)$ \\
\hline Emploi couvert par une convention collective & 0,072 & $(0,210)$ & 0,019 & $(0,753)$ & $-0,134$ & $(0,042)$ \\
\hline Emploi de supervision & & & & & 0,295 & $(0,000)$ \\
\hline Procédure d'évaluation du rendement & 0,390 & $(0,000)$ & 0,152 & $(0,002)$ & 0,084 & $(0,258)$ \\
\hline Utilisation d'un ordinateur & 0,217 & $(0,000)$ & 0,335 & $(0,000)$ & & \\
\hline \multicolumn{7}{|c|}{ Heures travaillées (Réf. $=$ entre 30 et 50 heures par semaine) } \\
\hline Moins de 30 heures par semaine & 0,012 & $(0,836)$ & $-0,014$ & $(0,830)$ & $-0,148$ & $(0,029)$ \\
\hline Plus de 50 heures par semaine & 0,008 & $(0,944)$ & 0,076 & $(0,702)$ & $-0,055$ & $(0,758)$ \\
\hline \multicolumn{7}{|l|}{ Niveau de scolarité(Réf. $=$ Pas de diplôme $)$} \\
\hline Diplôme d'études secondaires & 0,261 & $(0,007)$ & 0,007 & $(0,944)$ & 0,051 & $(0,603)$ \\
\hline Certificat & 0,291 & $(0,002)$ & 0,123 & $(0,217)$ & 0,012 & $(0,910)$ \\
\hline Diplôme collégial & 0,270 & $(0,003)$ & 0,025 & $(0,784)$ & 0,159 & $(0,112)$ \\
\hline Diplôme universitaire & 0,255 & $(0,007)$ & 0,089 & $(0,374)$ & $-0,014$ & $(0,895)$ \\
\hline \multicolumn{7}{|l|}{ Profession (Réf. = Personnel technique/métiers) } \\
\hline Gestionnaires & 0,026 & $(0,748)$ & 0,082 & $(0,308)$ & $-0,055$ & $(0,518)$ \\
\hline Professionnels & 0,120 & $(0,080)$ & 0,183 & $(0,014)$ & 0,006 & $(0,937)$ \\
\hline Commercialisation ou ventes & $-0,036$ & $(0,756)$ & $-0,069$ & $(0,589)$ & $-0,208$ & $(0,092)$ \\
\hline Personnel de bureau & $-0,208$ & $(0,007)$ & 0,012 & $(0,876)$ & $-0,036$ & $(0,650)$ \\
\hline Personnel non qualifié & $-0,424$ & $(0,000)$ & 0,196 & $(0,104)$ & $-0,028$ & $(0,827)$ \\
\hline Formation structurée & & & & & 0,039 & $(0,897)$ \\
\hline Formation structurée*sexe & & & & & 0,007 & $(0,941)$ \\
\hline Formation en cours d'emploi & & & & & 0,821 & $(0,010)$ \\
\hline Formation en cours d'emploi*sexe & & & & & 0,193 & $(0,046)$ \\
\hline
\end{tabular}

TAB. 5: Probabilité D’ OBTENIR UNE FORMATION STRUCTURÉE, UNE FORMATION EN COURS D'EMPLOI ET UNE PROMOTION

\footnotetext{
${ }^{6}$ En revanche, il n'y a pas de différence salariale significative entre les établissements à but lucratif et à but non lucratif.
} 


\begin{tabular}{|c|c|c|c|c|c|c|}
\hline \multirow[b]{2}{*}{ Variables } & \multicolumn{2}{|c|}{$\begin{array}{l}\text { FORMATION } \\
\text { STRUCTURÉE }\end{array}$} & \multicolumn{2}{|c|}{$\begin{array}{c}\text { FORMATION } \\
\text { EN COURS D'EMPLOI }\end{array}$} & \multicolumn{2}{|c|}{ PROMOTION } \\
\hline & Param. & P. value & Param. & P. value & Param. & P. value \\
\hline Etablissement à but non lucratif & $-0,136$ & $(0,159)$ & $-0,005$ & $(0,949)$ & $-0,041$ & $(0,656)$ \\
\hline Taux de roulement & $-0,015$ & $(0,570)$ & 0,058 & $(0,080)$ & 0,058 & $(0,043)$ \\
\hline Taux de roulement au carré & 0,000 & $(0,546)$ & $-0,000$ & $(0,128)$ & $-0,002$ & $(0,037)$ \\
\hline Proportion d'emplois vacants & 0,906 & $(0,385)$ & 1,576 & $(0,083)$ & $-1,145$ & $(0,240)$ \\
\hline Proportion d'emplois vacants au carré & $-1,723$ & $(0,347)$ & $-2,352$ & $(0,204)$ & 3,265 & $(0,082)$ \\
\hline \multicolumn{7}{|l|}{ Branche d'activité (Réf. = commerce de détail) } \\
\hline Exploitation des ressources naturelles & 0,354 & $(0,003)$ & $-0,069$ & $(0,574)$ & $-0,059$ & $(0,642)$ \\
\hline Industries de la fabrication & $-0,019$ & $(0,827)$ & $-0,033$ & $(0,732)$ & $-0,077$ & $(0,410)$ \\
\hline Constructions & 0,248 & $(0,030)$ & $-0,122$ & $(0,329)$ & 0,106 & $(0,347)$ \\
\hline Transport, entreposage et commerce de gros & 0,187 & $(0,061)$ & $-0,014$ & $(0,898)$ & 0,025 & $(0,813)$ \\
\hline Communications et autres services publics & 0,511 & $(0,000)$ & $-0,143$ & $(0,264)$ & 0,082 & $(0,503)$ \\
\hline Finance et assurances & 0,540 & $(0,000)$ & 0,163 & $(0,139)$ & 0,018 & $(0,890)$ \\
\hline Services immobiliers et de location & $-0,044$ & $(0,728)$ & 0,001 & $(0,994)$ & $-0,033$ & $(0,808)$ \\
\hline Services aux entreprises & 0,147 & $(0,168)$ & $-0,040$ & $(0,747)$ & 0,136 & $(0,260)$ \\
\hline Enseignement et services de soins de santé & 0,339 & $(0,006)$ & $-0,123$ & $(0,261)$ & $-0,271$ & $(0,028)$ \\
\hline Information et industries culturelles & 0,083 & $(0,469)$ & $-0,061$ & $(0,611)$ & $-0,001$ & $(0,994)$ \\
\hline \multicolumn{7}{|c|}{ Taille de l'établissement (Réf. = 500 employés et plus) } \\
\hline Moins de 20 employés & $-0,479$ & $(0,000)$ & $-0,247$ & $(0,003)$ & $-0,073$ & $(0,476)$ \\
\hline Entre 20 et 99 employés & $-0,192$ & $(0,003)$ & 0,009 & $(0,899)$ & $-0,001$ & $(0,987)$ \\
\hline Entre 100 et 499 employés & $-0,006$ & $(0,909)$ & $-0,149$ & $(0,021)$ & 0,004 & $(0,951)$ \\
\hline \multicolumn{7}{|l|}{ Région (Réf. = Ontario) } \\
\hline Colombie-Britannique & $-0,013$ & $(0,852)$ & 0,043 & $(0,520)$ & $-0,045$ & $(0,508)$ \\
\hline Alberta & $-0,104$ & $(0,139)$ & 0,117 & $(0,131)$ & 0,029 & $(0,718)$ \\
\hline Québec & $-0,002$ & $(0,967)$ & $-0,513$ & $(0,000)$ & 0,118 & $(0,107)$ \\
\hline Provinces de l'Atlantique & 0,022 & $(0,760)$ & $-0,195$ & $(0,010)$ & 0,101 & $(0,226)$ \\
\hline Provinces des Prairies & 0,008 & $(0,915)$ & 0,088 & $(0,271)$ & $-0,124$ & $(0,178)$ \\
\hline$\rho_{\text {se }}$ & & 0,096 & & & 002) & \\
\hline$\rho_{s p}$ & & 0,040 & & & 822) & \\
\hline$\rho_{e p}$ & & $-0,385$ & & & 032) & \\
\hline Nbre d'observations & & & & & & \\
\hline
\end{tabular}

TAB. 5: Probabilité D’obTeniR UNE FORMATION STRUCTURÉE, UNE FORMATION EN COURS D'EMPLOI ET UNE PROMOTION (SUITE)

Le reste de nos commentaires se concentrera sur les trois questions qui nous intéressent particulièrement, à savoir : 1) les liens entre les deux types de formation continue ;2) les différences de rendement existant entre formation professionnelle formelle et informelle ; 3) la valorisation différenciée entre hommes et femmes des formations et des promotions. 


\begin{tabular}{lcc}
\hline Variables & Paramètre & P. value \\
\hline Constante & 1,900 & $(0,000)$ \\
Sexe (homme=1) & 0,145 & $(0,000)$ \\
Probabilité de suivre une formation structurée & 0,394 & $(0,000)$ \\
Probabilité de suivre une formation structurée*sexe & $-0,055$ & $(0,535)$ \\
Probabilité de suivre une formation en cours d'emploi & 0,264 & $(0,072)$ \\
Probabilité de suivre une formation en cours d'emploi*sexe & 0,041 & $(0,751)$ \\
Probabilité d'obtenir une promotion & 0,159 & $(0,004)$ \\
Probabilité d'obtenir une promotion*sexe & 0,016 & $(0,831)$ \\
Marié & 0,084 & $(0,000)$ \\
En couple & 0,084 & $(0,000)$ \\
Enfants à charges & 0,000 & $(0,998)$ \\
Ancienneté avec l'employeur & 0,001 & $(0,000)$ \\
Ancienneté au carré /100 & $-0,000$ & $(0,110)$ \\
Expérience à temps plein & 0,019 & $(0,000)$ \\
Expérience au carré/100 & $-0,032$ & $(0,000)$ \\
& & \\
Niveau de scolarité (Réf. = Pas de diplômes d'études secondaires) & & \\
$\quad$ Diplômes d'études secondaires & 0,076 & $(0,000)$ \\
$\quad$ Certificat & 0,108 & $(0,000)$ \\
$\quad$ Diplôme collégial & 0,118 & $(0,000)$ \\
Diplôme universitaire & 0,259 & $(0,000)$ \\
& & \\
Heures travaillées & & \\
Temps partiel & 0,017 & $(0,324)$ \\
$\quad$ Horaire de travail flexible & 0,014 & $(0,259)$ \\
$\quad$ Travail entre 6h et 18h & $-0,013$ & $(0,444)$ \\
$\quad$ Heures habituelles non rémunérées & 0,008 & $(0,000)$ \\
Statut d'emploi & & \\
$\quad$ Emploi permanent & & \\
$\quad$ Emploi de supervision & 0,002 & $(0,926)$ \\
$\quad$ Emploi syndiqué & 0,042 & $(0,001)$ \\
Procédure d'évaluation du rendement & 0,049 & $(0,002)$ \\
& $-0,018$ & $(0,186)$ \\
\hline
\end{tabular}

TAB. 6: EQUATION DE SALAIRE POUR LES EMPLOIS OCCUPÉS EN 2000 : INFLUENCE DES FORMATIONS STRUCTURÉE ET INFORMELLE

\subsection{Les déterminants des formations structurée et en cours d'emploi}

Le premier résultat à souligner est que le coefficient de corrélation $\rho_{s e}$ entre les deux équations de participation à une formation est statistiquement significatif. Certaines caractéristiques individuelles inobservables jouent simultanément sur les chances du salarié de suivre une formation structurée et sur celles de suivre une formation en cours d'emploi. Cela laisse penser que la probabilité de suivre une formation structurée influence positivement la probabilité de suivre une formation en cours d'emploi durant la même période et inversement. Ainsi, des liens de complémentarité existeraient entre les deux types de formation continue, reflétant par exemple une décision commune mais inobservée d'investir en capital humain. Il était donc indispensable d'estimer simultanément les équations de participation via un probit multivarié.

Les taux de participation à une formation structurée et en cours d'emploi sont croissants avec la taille de l'établissement, l'utilisation d'un ordinateur par le salarié et l'existence d'une procédure d'évaluation courante de son rendement. Les travailleurs dont le rendement est évalué par une procédure 


\begin{tabular}{lcc}
\hline Variables & Paramètre & P. value \\
\hline Etablissement à but non lucratif & $-0,007$ & $(0,760)$ \\
Etablissement à propriété étrangère & 0,064 & $(0,002)$ \\
& & \\
Profession (Réf. = Personnel technique/métiers) & & \\
$\quad$ Gestionnaires & 0,187 & $(0,000)$ \\
$\quad$ Professionnels & 0,176 & $(0,000)$ \\
Commercialisation ou ventes & $-0,092$ & $(0,003)$ \\
Personnel de bureau & $-0,077$ & $(0,000)$ \\
Personnel non qualifié & $-0,109$ & $(0,000)$ \\
& & \\
Branche d'activité (Réf. $=$ Commerce de détail) & & \\
Exploitation des ressources naturelles & 0,397 & $(0,000)$ \\
Industries de la fabrication & 0,245 & $(0,000)$ \\
Constructions & 0,359 & $(0,000)$ \\
Transport, entreposage et commerce de gros & 0,233 & $(0,000)$ \\
Communications et autres services publics & 0,270 & $(0,000)$ \\
Finance et assurances & 0,223 & $(0,000)$ \\
Services immobiliers et de location & 0,274 & $(0,000)$ \\
Services aux entreprises & 0,251 & $(0,000)$ \\
Enseignement et services de soins de santé & 0,213 & $(0,000)$ \\
Information et industries culturelles & 0,264 & $(0,000)$ \\
& & \\
Taille de l'établissement (Réf. = 500 employés et plus) & \\
Moins de 20 employés & $-0,124$ & $(0,000)$ \\
Entre 20 et 99 employés & $-0,147$ & $(0,000)$ \\
Entre 100 et 499 employés & $-0,078$ & $(0,000)$ \\
Région (Réf. = Ontario) & & \\
Colombie-Britannique & & \\
Alberta & 0,024 & $(0,282)$ \\
Provinces des Prairies & $-0,090$ & $(0,000)$ \\
Québec & $-0,153$ & $(0,000)$ \\
Provinces de l'Atlantique & $-0,036$ & $(0,174)$ \\
\hline & $-0,223$ & $(0,000)$ \\
\hline
\end{tabular}

TAB. 6: EQUATION DE SALAIRE POUR LES EMPLOIS OCCUPÉS EN 2000 : INFLUENCE DE LA FORMATION STRUCTURÉE ET EN COURS D'EMPLOI (SUITE)

courante (rapport écrit, rencontre personnelle, rapport normalisé) anticipent vraisemblablement que leur acquisition de nouvelles connaissances aura plus de chances d'être reconnue par son employeur et donc d'être récompensée. En revanche, contrairement à certains résultats antérieurs de la littérature, l'expérience professionnelle, l'ancienneté, le nombre d'heures travaillées et le caractère permanent de l'emploi n'influencent pas significativement la participation aux deux types de formation en entreprise. Premièrement, cela suggère que la formation professionnelle au Canada est un réel processus continu qui se développe, de manière ininterrompue, tout au long de la vie active : l'acquisition et la mise à jour de toutes sortes de capacités, d'intérêts, de connaissances et de qualifications auraient lieu au fur et à mesure du déroulement des carrières. Deuxièmement, il n'y aurait pas au Canada d'inégalités d'accès aux formations continues en fonction du statut de l'emploi occupé : les salariés travaillant à temps partiel et/ou dans un emploi temporaire ont, toutes choses étant égales par ailleurs, un taux de participation aux formations en entreprise similaires aux salariés à temps complet et/ou occupant un emploi permanent. 
La différence la plus marquée entre les deux types de formation continue est que le niveau de scolarité a une forte influence sur la participation à une formation structurée mais aucune sur celle à une formation en cours d'emploi. La formation formelle dispensée par les entreprises est complémentaire à celle dispensée par le système scolaire. Ainsi, elle permet peu aux salariés non diplômés et non qualifiés de rattraper leur retard. A l'inverse, la région du siège social de l'établissement n'a une incidence que sur la formation en cours d'emploi. Toutes les provinces auraient des taux de formation structurée similaires; en revanche, le Québec et les Provinces de l'Atlantique accuseraient un net retard dans la formation informelle, toutes choses étant égales par ailleurs.

\subsection{Des écarts de rendement entre formation formelle et formation informelle}

Regardons maintenant s'il existe une différence dans les rendements de ces deux formes de formation en entreprise, en termes d'accession à un poste à plus hautes responsabilités et en termes d'accroissement du niveau des rémunérations.

La troisième colonne du tableau 5 montre que seule la formation en cours d'emploi est un atout pour obtenir une promotion la même année. Toutes choses étant égales par ailleurs, les salariés ayant suivi une formation en cours d'emploi ont plus de chances d'être promus que les salariés ayant suivi une formation structurée, ce qui confirme les résultats du tableau 3. On peut supposer que la formation informelle soit davantage axée sur les tâches spécifiques d'un emploi tandis que les compétences acquises lors de cours structurés seraient plus générales. Il n'est pas alors déraisonnable de penser que la formation informelle accroît les chances de promotion dans l'année alors que la formation formelle, plus longue, aura davantage un impact sur les chances de promotion à plus long terme. La non-significativité du coefficient de corrélation, $\rho_{s p}$, va dans le sens de la non-simultanéité des décisions de formation structurée et de promotion.

La corrélation négative entre les résidus des équations de formation informelle et de promotion, $\rho_{e p}$, peut paraître plus surprenante vu que le coefficient associé à la variable de "formation en cours d'emploi" est significatif et positif dans l'équation de promotion. Néanmoins, ce résultat s'explique par la double orientation de la formation informelle. Elle peut en effet être axée soit sur les tâches de l'emploi actuel, soit sur celles d'un éventuel emploi futur. La corrélation négative signifie que des caractéristiques individuelles inobservables qui influencent positivement la formation en cours d'emploi, influencent aussi négativement les chances de promotion. On peut par exemple penser à un manque d'autonomie de certains salariés dans leur emploi actuel qui demandent l'aide de leurs collègues - ce qui prend la forme d'une formation informelle - pour leur expliquer certaines tâches de leur emploi. N'ayant pas assimiler les bases de leur poste, ces salariés ont peu de chances d'être promus. En revanche, les salariés qui suivent une formation informelle afin d'élargir le champ de compétences non requises par leur poste, ont une probabilité plus élevée d'obtenir une promotion que ceux qui n'en suivent pas. Ainsi, la formation en cours d'emploi serait bien un atout pour obtenir une promotion, à moins qu'elle ne dénote et vise à combler, comme dans certains cas plus rares, un manque d'autonomie ou de compétences dans l'emploi actuel.

Selon le tableau 6, la participation à une formation structurée et celle à une formation en cours d'emploi ont une incidence positive et significative sur le niveau des rémunérations. Toutefois, la formation informelle a un rendement salarial plus faible que la formation formelle. Cela suggère que lorsque l'employeur contrôle de très près le contenu de la formation professionnelle, il y a de 
fortes chances que les connaissances acquises par le salarié augmentent sa productivité et par voie de conséquence qu'il se voit offrir des augmentations de salaire. En revanche, l'accroissement de productivité individuelle engendré par une formation en cours d'emploi est sans doute plus ardu à mesurer et donc moins valorisé financièrement.

En résumé, la formation continue structurée aurait au Canada uniquement une incidence directe sur les salaires, alors que la formation continue informelle aurait non seulement une incidence directe mais aussi une incidence indirecte via les promotions, qui elles influencent positivement le niveau de rémunération individuelle. Toutefois, le rendement salarial d'une formation structurée est significativement plus élevé que celui d'une promotion, toutes choses égales par ailleurs. D'un point de vue salarial, une formation structurée serait aussi payante qu'une formation en cours d'emploi qui se solde par une promotion.

\subsection{Des taux de formations et des valorisations similaires entre hommes et femmes}

Il nous reste à regarder si les hommes et les femmes ont un accès différencié à la formation continue et si celle-ci joue le même rôle pour les deux sexes en termes de salaires et de promotions.

Les variables de sexe dans le tableau 5 ne sont pas statistiquement significatives, indiquant qu'à caractéristiques individuelles et professionnelles similaires, les hommes et les femmes ont des taux moyens identiques de participation aux formations continues et d'obtention d'une promotion. Nos résultats confirment ceux de Turcotte et al. (2003) sur les formations en entreprise et ceux de Booth et al. (1998) sur les promotions. Or, la richesse du double questionnaire employeur/employé de l'EMTE a permis de retenir comme déterminants des décisions de formations et de promotions, des variables habituellement non prises en compte simultanément car non renseignées. Ainsi, l'accès différencié à la formation continue mis en évidence dans certaines études pourrait simplement refléter des différences hommes/femmes dans des caractéristiques non observées (ex : procédure d'évaluation, utilisation d'un ordinateur, taux de roulement et d'emplois vacants) que de réels écarts dans le taux de formation, toutes choses étant égales par ailleurs.

Une formation en cours d'emploi se concrétiserait plus souvent en une promotion pour les hommes que pour les femmes - la variable formation informelle ${ }^{*}$ sexe est statistiquement significative dans l'équation caractérisant le processus de promotion. Les femmes suivant une formation informelle auraient ainsi plus de difficultés à faire reconnaître par leurs employeurs leur acquisition de compétences et donc à progresser dans la hiérarchie. Cela suggère que plus un investissement en formation est imparfaitement observé ou contrôlé, plus les employeurs peuvent être enclins à se baser sur des préjugés ou autres critères, défavorables à la promotion des femmes.

Néanmoins, les rendements salariaux des deux types de formation continue sont identiques entre hommes et femmes au Canada. De même, une promotion génère, toutes choses étant égales par ailleurs, les mêmes accroissements de salaire pour les deux sexes. Les femmes suivant une formation informelle auraient ainsi moins de chances que les hommes d'obtenir une promotion, mais celles qui ont l'opportunité d'en recevoir une, peuvent s'attendre à une augmentation de salaire comparable à celle de leurs homologues masculins.

Les différences d'accès aux promotions à la suite d'une formation en cours d'emploi peuvent se traduire par un écart salarial entre sexes puisque les promotions agissent sur les salaires. En revanche, 
les pratiques des employeurs en termes de valorisations salariales des formations continues et des promotions ne semblent pouvoir être remises en cause du point de vue de l'égalité hommes/femmes. Toutefois, au Canada, les femmes ont, en moyenne, à caractéristiques individuelles et professionnelles comparables, des rémunérations inférieures de $13,5 \%$ à celles des hommes.

\section{Conclusion}

Cet article propose une étude de l'impact des actions de formation en entreprise sur les salaires et les perspectives professionnelles des salariés canadiens, qui détaille les différences de valorisation entre sexes. Son originalité est de distinguer les formations continues formelle et informelle, ce qui offre la possibilité d'examiner les liens entre elles, de comparer leurs influences respectives sur les carrières et de savoir s'il existe des différences entre hommes et femmes dans la nature et les rendements des formations suivies. Jusqu'à présent, les études de rentabilité sur la formation continue s'étaient concentrées sur la formation formelle - faute de données disponibles sur la formation informelle - et sur les augmentations salariales, négligeant son impact en termes de promotions.

Ici, dans une première étape, on estime, par maximum de vraisemblance simulé, un probit trivarié récursif afin de modéliser simultanément la probabilité de suivre les différentes formations en entreprise et leur influence sur l'obtention d'une promotion. Dans une deuxième étape, les rendements salariaux des formations et des promotions sont évalués à l'aide d'équations de salaires où les probabilités prédites par le probit trivarié récursif sont utilisées comme instruments.

Les résultats obtenus avec l'Enquête sur le milieu du travail et les employés (EMTE) 1999-2000 montrent que la formation en entreprise est un élément prépondérant dans les carrières individuelles. Ils confirment que les décisions conduisant à former un salarié et à le promouvoir se prennent souvent de manière concomitante. D'où la nécessité d'utiliser des techniques économétriques tenant compte de ces interdépendances. D'un point de vue pratique, il apparaît aussi indispensable de distinguer les différents types de formation en entreprise dans les estimations de rentabilité. La formation formelle et informelle ont des impacts bien distincts en termes d'augmentations salariales et de promotions. La formation structurée a une action directe sur les salaires mais pas sur les promotions. Au contraire, la formation informelle joue non seulement sur les salaires de façon directe mais aussi sur les promotions et donc de façon indirecte sur les salaires. Au final, la formation structurée a une rentabilité plus élevée que la formation informelle, à moins que celle-ci ne se solde par une promotion.

Du point de vue des disparités professionnelles entre sexes, cette étude nous dresse un tableau plutôt encourageant pour le Canada. D'une part, les hommes et les femmes ont des taux d'accès similaires pour les deux types de formation continue. D' autre part, la formation formelle a exactement la même valorisation professionnelle et salariale pour les deux sexes. En revanche, la formation informelle a une meilleure rentabilité en termes de promotions pour les hommes. En conséquence, il serait davantage recommandé de développer des cours de formation structurée que des formations informelles afin de limiter les disparités salariales entre sexes. Plus le contenu de la formation proposée est précis et plus les compétences acquises et les progrès individuels peuvent être facilement évalués, plus l'accroissement de productivité a des chances d'être reconnu et récompensé en termes de salaires, car les employeurs se basent alors davantage sur des critères objectifs que des préjugés ou autres critères défavorables aux femmes. 
On a aussi montré que les travailleurs ayant une formation initiale la plus avancée sont les plus susceptibles de suivre une formation structurée. Or actuellement, on observe au Canada, que les succès scolaires et universitaires des jeunes femmes sont supérieures à ceux de leurs homologues masculins. On peut donc être optimiste pour l'avenir d'autant plus que la spécialisation sexuée des études commence à être remise en cause. Par exemple, les femmes sont majoritaires dans les départements de médecine, pharmacie ou droit des écoles professionnelles et leur nombre est en constante progression dans les facultés de génie. Il paraît ainsi indispensable de continuer à élargir les choix professionnels des femmes par une évolution de l'orientation scolaire. Une fois qu'elles auront accès aux mêmes types d'emploi, les progressions de carrière hommes/femmes devraient être comparables à en croire les valorisations professionnelles et salariales que l'on a trouvé.

\section{Références}

Aitken, B., Harrison, A. \& Lipsey, R. (1996), 'Wages and Foreign Ownership : A Comparative Study of Mexico, Venezuela and the United States', Journal of International Economics 40(3/4), 345371.

Becker, G. (1964), Human Capital. A Theoretical and Empirical Analysis with Special Reference to Education, New York and London : Columbia University Press.

Béret, P. \& Dupray, A. (1998), 'La formation professionnelle continue : de l'accumulation de compétences à la validation de la performance', Formation-Emploi 63(Septembre), 61-80.

Booth, A., Francesconi, M. \& Frank, J. (1998), 'Glass Ceilings or Sticky Floors ?', Center for Economic Policy Research Discussion Paper 1965(September).

Cabral, R., Ferber, M. \& Green, C. (1981), 'Men and Women in Fiduciary Institutions : A Study of Sex Differences in Career Development', Review of Economics and Statistics 63(4), 573-580.

Cannings, K. (1988), 'Managerial Promotion : The Effects of Socialization, Specialization, and Gender', Industrial and Labor Relations Review 42(1), 77-88.

Cobb-Clark, D. (2001), Getting Ahead : The Determinants of and Payoffs to Internal Promotion for Young U.S Men and Women, in S. Polachek, ed., 'Research in Labor Economics : Worker Wellbeing in a Changing Labor Market', Vol. 20, JAI, Elsevier Science, pp. 339-372.

Corcoran, M. \& Duncan, G. (1979), 'Work History, Labor Force Attachment, and Earnings Differences Between the Sexes', Journal of Human Resources 14(1), 3-20.

Delame, E. \& Kramarz, F. (1997), 'Entreprises et formation continue', Economie et Prévision 127, 6382.

DiPrete, T. \& Soule, W. (1988), 'Gender and Promotion in Segmented Job Ladder Systems', American Sociological Review 53(1), 26-40.

Drolet, M. (2002), 'New Evidence on Canadian Gender Pay Differentials : Does Measurement Matter?', Canadian Public Policy 28(1).

Duncan, G. \& Hoffman, S. (1979), 'On-the-Job Training and Earnings Differences by Race and Sex', Review of Economics and Statistics 61(4), 593-603.

Feliciano, Z. \& Lipsey, R. (1999), 'Foreign Ownership and Wages in the United States, 1987-1992', NBER Working Paper (6923). 
Gauron, A. (2000), Formation tout au long de la vie, in 'Rapport du Conseil d'Analyse économique', Vol. 22, La documentation française : Paris.

Gerhart, B. \& Milkovich, G. (1989), Salaries, Salary Growth, and Promotions of Men and Women in a Large, Private Firm, in R. Michael, H. Hartmann \& B. O'Farrell, eds, 'Pay Equity : Empirical Inquiries', National Academy Press, Washington D.C, pp. 23-41.

Geweke, J., Keane, M. \& Runkle, D. (1994), 'Alternative Computational Approaches to Inference in the Multinomial Probit Model', The Review of Economics and Statistics LXXXVI(4), 609-632.

Greene, W. (2003), Econometric Analysis, 5ème édition, Prentice Hall.

Gronau, R. (1988), 'Sex-Related Wage Differentials and Women's Interrupted Careers - the Chicken or the Egg ?', Journal of Labor Economics 6(3), 277-301.

Groot, W. \& Maassen von den Brink, H. (1996), 'Glass Ceilings or Dead Ends : Job Promotion of Men and Women Compared', Economics Letters 53(2), 221-226.

Handy, F. \& Katz, E. (1998), 'The Wage Differential between Non-Profit Institutions and Corporations : Getting More by Paying Less ?', Journal of Comparative Economics 26(2), 246-261.

Hartman, H. (1987), Internal Labor Markets and Gender : A Case Study of Promotion, in C. Brown \& J. Pechman, eds, 'Gender in the Workplace', The Brookings Institution, Washington D.C, pp. 59-97.

Hersch, J. \& Viscusi, K. (1996), 'Gender Differences in Promotions and Wages', Industrial Relations 35(4), 461-472.

Hill, E. (2001), 'Post-School-Age Training among Women : Training Methods and Labor Market Outcomes at Older Ages', Economics of Education Review 20(2), 181-191.

Laband, D. \& Lentz, B. (1993), 'Is There Sex Discrimination in the Legal Profession ? Further Evidence on Tangible and Intangible Margins', Journal of Human Resources 28(2), 230-258.

Leete, L. (2001), 'Whither the Non-Profit Wage Differential ? Estimates from the 1990 Census', Journal of Labor Economics 10(1), 136-170.

Lewis, G. (1986), 'Gender and Promotions : Promotion Chances of White Men and Women in Federal White-Collar Employment', Journal of Human Resources 21(3), 406-419.

Lillard, L. \& Tan, H. (1992), Private Sector Training : Who Gets It and What Are Its Effects ?, in R. Ehrenberg, ed., 'Reserach in Labor Economics', Vol. 13, Grennwich, Conn. and London : JAI Press, pp. 1-62.

Lipsey, R. (1994), 'Foreign-Owned Firms and U.S. Wages', NBER Working Paper (4927).

Long, S., Allison, P. \& McGinnis, R. (1993), 'Rank Advancement in Academic Careers : Sex Differences and the Effects of Productivity', American Sociological Review 58(5), 703-722.

Lynch, L. (1992), 'Private-Sector Training and the Earnings of Young Workers', American Economic Review 82(1), 299-312.

Maddala, G. (1983), Limited Dependent and Qualitative Variables in Econometrics, Cambridge University Press.

McCue, K. (1996), 'Promotions and Wage Growth', Journal of Labor Economics 14(2), 175-209.

Michaudon, H. (2000), 'Investir dans la formation continue', Insee Première 697(Février), 4 pages.

Mincer, J. (1974), Schooling, Experience and Earnings, Columbia University Press for NBER, New York. 
OCDE (1995), L'étude de l'OCDE sur l'emploi, Partie II : possibilités d'adaptation des marchés du travail, OCDE, Paris.

Olsen, R. \& Sexton, E. (1996), 'Gender Differences in the Returns to and the Acquisition of On-theJob Training', Industrial Relations 35(1), 59-77.

Olson, C. \& Becker, B. (1983), 'Sex Discrimination in the Promotion Process', Industrial and Labor Relations Review 36(4), 624-641.

Pargamit, M. \& Veum, J. (1999), 'What is a Promotion?', Industrial and Labor Relations Review 52(4), 581-601.

Paulin, E. \& Mellor, J. (1996), 'Gender, Race and Promotion Within a Private Sector Firm', Industrial Relations 35(2), 276-295.

Powell, G. \& Butterfield, A. (1994), 'Investigating the "Glass Ceiling” Phenomenon : An Empirical Study of Actual Promotions to Top Management', Academy Management Journal 37(1), 1994.

Pudney, S. \& Shields, M. (2000), 'Gender and Racial Discrimination Pay and Promotion for NHS Nurses', Oxford Bulletin of Economic and Statistics 62(0), 801-835. Special Issue.

Royalty, A. (1996), 'The Effects of Job Turnover on the Training of Men and Women', Industrial and Labor Relations Review 49(3), 506-521.

Ruhm, C. \& Borkoski, C. (2001), 'Compensation in the Non-Profit Sector', Compensation in the Non-Profit Sector (7562).

Shenhav, Y. (1992), 'Entrance of Blacks and Women into Managerial Positions in Scientific and Engineering Occupations : A Longitudinal Analysis', Academy of Management Journal 35(4), 889901.

Spurr, S. (1990), 'Sex Discrimination in the Legal Profession : A Study of Promotion', Industrial and Labor Relations Review 43(4), 406-417.

Spurr, S. \& Sueyoshi, G. (1994), 'Turnover and Promotion of Lawyers : An Inquiry into Gender Differences', Journal of Human Resources 29(3), 813-842.

Stern, S. (2000), Simulation-Based Inference in Econometrics : Motivation and Methods, in R. Mariano, T. Schuermann \& M. Weeks, eds, 'Simulation-Based Inference in Econometrics : Methods and Applications', Cambridge, pp. 9-37.

Stewart, L. \& Gudykunst, W. (1982), 'Differential Factors Influencing the Hierarchical Level and Number of Promotion of Males and Females Within an Organization', Academy of Management Journal 25(3), 586-597.

Turcotte, J., Léonard, A. \& Montmarquette, C. (2003), 'Nouveaux résultats sur les déterminants de la formation dans les emplacements canadiens', La série sur le milieu de travail en évolution, Statistique Canada 71-584-MIE(5).

Veum, J. (1996), 'Gender and Race Differences in Company Training', Industrial Relations 35(1), 3244. 